\title{
Effect of intravenous thrombolysis with alteplase on clinical efficacy, inflammatory factors, and neurological function in patients with acute cerebral infarction
}

\author{
Jinhua Wang ${ }^{1 \oplus \otimes}$, Xia Fang ${ }^{2 \oplus}$, Dongliang Wang ${ }^{1 \oplus}$, and Yuan Xiao ${ }^{1 \oplus}$ \\ ${ }^{1}$ Department of Neurology, The People's Hospital of Beilun District, Beilun Branch Hospital of The First Affiliated Hospital, \\ Zhejiang University School of Medicine, Ningbo, Zhejiang Province, China \\ ${ }^{2}$ Department of Gynecology, The People's Hospital of Beilun District, Beilun Branch Hospital of The First Affiliated Hospital, \\ Zhejiang University School of Medicine, Ningbo, Zhejiang Province, China
}

\begin{abstract}
This study aimed to explore the effect of intravenous thrombolysis with alteplase on clinical efficacy, inflammatory factors, and neurological function in patients with acute cerebral infarction. A total of 120 patients with acute cerebral infarction were divided into two groups by the random number table method, with 60 patients in each group: observation group (intravenous thrombolysis with alteplase) and control group (intravenous thrombolysis with batroxobin). The clinical efficacy after a 14-day treatment was observed. Serum C-reactive protein (CRP), tumor necrosis factor $\alpha$ (TNF- $\alpha$ ), interleukin-6 (IL-6), CD62p, GMP140, and neuron-specific enolase (NSE) were measured. Scores of National Institutes of Health Stroke Scale (NIHSS), MiniMental State Examination (MMSE), and Montreal Cognitive Assessment (MoCA) were determined. The total effective rate in the observation group was $81.67 \%$, which was higher than the $61.67 \%$ in the control group $(P<0.05)$. The improvement of inflammatory factors (CRP, TNF- $\alpha$, IL-6, CD62p, GMP-140, and NSE), NIHSS, MMSE, and MoCA in the observation group was superior to that in the control group (all $\mathrm{P}<0.05$ ). The modified Rankin scale at three months after hospital discharge in the observation group was lower than that in the control group $(P<0.01)$. Intravenous thrombolysis with alteplase for acute cerebral infarction can enhance the clinical efficacy, alleviate inflammatory response and brain injury, and improve cognitive function, which is worthy of further clinical application and study.
\end{abstract}

\section{Introduction}

Acute cerebral infarction $(\mathrm{ACl})$ is caused by the stenosis or occlusion of the artery feeding the brain, inducing insufficient cerebral blood supply, which leads to cerebral ischemia and hypoxia and causes brain injury and necrosis, eventually damaging brain function $(1,2)$. The incidence of $\mathrm{ACl}$ rises year by year (3), and $30 \%$ of patients are the elderly (4-6). ACl is the most common cause of death in China (7). Early thrombolytic therapy for ACI may reduce the degree of injury to the brain (8). Studies have proven the thrombolysis time window for $\mathrm{ACl}$ is within $4.5 \mathrm{~h}(9,10)$. The thrombolytic drug batroxobin was recommended by the 2014 Guidelines for Diagnosis and Treatment of Acute Ischemic Stroke in China, which has been widely applied in early ACl patients in China with remarkable clinical efficacy (11). In recent years, alteplase, the second generation of thrombolytic drugs, has been applied in clinical practice and is recommended for the treatment of $\mathrm{ACl}$ according to evidence-based medicine in Europe and North America. Previous studies found that $\mathrm{ACl}$ patients who were evaluated by the National Institute of Neurological Disorders and Stroke (NINDS) criteria could have significant benefits from alteplase and the administration should be as early as possible $(12,13)$. However, alteplase has not been widely used in China because of its high price. Therefore, the advantages and disadvantages of batroxobin and alteplase in thrombolysis effect have not been determined and compared in China (14).

A study revealed that inflammatory response is one of the important pathologic changes of $\mathrm{ACl}$ and plays a vital role in thrombosis and nerve function deficit. Abnormal high levels of inflammatory factors can induce oxidative stress response, causing oxidative stress injury to the 
brain tissue (15). Neuron-specific enolase (NSE) level rapidly increases after early cell injury and is a sensitive indicator for clinical judgment of cell injury (16).

In this study, we investigated the therapeutic effects of batroxobin and alteplase through a comprehensive evaluation in terms of clinical efficacy, effect on inflammatory factors, and improvement of neurological function, expecting to provide more references for clinical practice.

\section{Material and Methods}

\section{Clinical data}

This study was approved by the Ethics Committee of The People's Hospital of Beilun District, Beilun Branch Hospital of The First Affiliated Hospital of Zhejiang University School of Medicine. A total of 120 patients with $\mathrm{ACl}$ were recruited from the Department of Neurology of The People's Hospital of Beilun District, Beilun Branch Hospital from January 2017 to June 2018 and they were divided into two groups by the random number table method, with 60 patients in each group: observation group (intravenous thrombolysis with alteplase) and control group (intravenous thrombolysis with batroxobin). All patients were over 65 years old, and all the patients enrolled in this study or their families signed the informed consent form.

\section{Inclusion criteria}

Patients met the diagnostic criteria for ACl of the 2014 Guidelines for Diagnosis and Treatment of Acute Ischemic Stroke in China (17): on hospital admission, $\mathrm{ACl}$ patients were identified by CT scans and MRI was performed whenever an alternative diagnosis was suspected; patients were 65-76 years old; patients were diagnosed with $\mathrm{ACl}$ for the first time; the time from onset to admission for intravenous thrombolysis was within $4.5 \mathrm{~h}$; the scores of National Institutes of Health Stroke Scale (NIHSS) were 4-15 at admission (18).

\section{Exclusion criteria}

Exclusion criteria were: patients allergic to alteplase or batroxobin; patients with a medical history of craniocerebral trauma, epilepsy, and cerebrovascular disease; patients who could not cooperate with cognitive function evaluation; patients who had coagulopathy or administration of heparin or oral anticoagulant drugs before treatment; patients with cardiopulmonary insufficiency; patients with malignant tumor; patients who had mental disorder that influenced cognition; patients who were in lactation or gestation periods; patients who had hemorrhage in the digestive tract or urinary tract; patients who could not finish the MiniMental State Examination (MMSE) and Montreal Cognitive Assessment (MoCA) within $30 \mathrm{~min}$ at admission.

\section{TOAST classification of stroke}

The TOAST classification of stroke according to Classification of Subtype of Acute Ischemic Stroke in 1993 was applied (19): atherothrombosis, stroke of undetermined etiology, cardioembolism, small-vessel occlusion, and stroke of other determined etiology.

\section{Methods}

Patients in the two groups were treated according to the treatment regimen of $\mathrm{ACl}$ described in the 2014 Guidelines for Diagnosis and Treatment of Acute Ischemic Stroke in China (17), including electrocardiogram monitoring of vital signs, oxygen inhalation, monitoring and controlling blood pressure and blood glucose, dehydration for releasing intracranial pressure, antiplatelet and anticoagulation therapy, the use of free-radical scavenger such as edaravone and other neuroprotective agents, and others. After vital signs were stable, the patients were guided to perform functional rehabilitation.

Patients in the observation group were given alteplase (Boehringer Ingelheim, Germany) at a total dosage of 0.9 $\mathrm{mg} / \mathrm{kg}$, in addition to general treatment. Ten percent of the total dosage was injected intravenously within $1 \mathrm{~min}$, and the remaining $90 \%$ was dripped intravenously over $1 \mathrm{~h}$. The course of treatment lasted 14 days.

Patients in the control group were given batroxobin (Beijing Tobishi Pharmaceutical Co., Ltd., China), in addition to general treatment. The initial dose on the first day of admission was 10 batroxobin units (BU), which were dripped intravenously over $1 \mathrm{~h}$, and on the third day, $5 \mathrm{BU}$ batroxobin was dripped intravenously over $1 \mathrm{~h}$. The course of treatment lasted 14 days.

\section{Outcome measures}

Primary outcome measures were: treatment efficacy, levels of inflammatory factors, and neurological impairment evaluation. Secondary outcome measures were: cognitive assessment, prognostic assessment, and adverse events.

Treatment efficacy after the 14-day treatment was evaluated according to the $\mathrm{ACl}$ criteria described in the 2014 Guidelines for Diagnosis and Treatment of Acute Ischemic Stroke in China (17): the efficacy was evaluated based on the degree of decline of NIHSS score before and after treatment. A decline of $91-100 \%$ indicated recovery; a decline of $46-90 \%$ indicated remarkable improvement; a decline of $18-45 \%$ indicated improvement; a decline of $\leqslant 17 \%$ indicated no change. Total effectiveness rate was calculated as (number of recovery cases + the number of remarkable improvement cases + the number of improvement cases)/total case number $\times 100 \%$. The degree of decline was calculated as (NIHSS score before treatment - NIHSS score after treatment)/NIHSS score before treatment $\times 100 \%$.

Venous blood $(5 \mathrm{~mL})$ was drawn at admission and at 8:00 am after the 14-day treatment to evaluate the levels of inflammatory factors of patients. The blood sample was stored in a refrigerator at $4^{\circ} \mathrm{C}$ for $15 \mathrm{~min}$. The serum and plasma were separated by centrifugation at $3,100 \mathrm{~g}$ for 
$10 \min \left(45^{\circ} \mathrm{C}\right)$. The plasma was collected and $40 \mu \mathrm{L}$ phosphate buffered saline containing protease inhibitor was added and this was then stored in a refrigerator at $-80^{\circ} \mathrm{C}$. Serum C-reactive protein (CRP), tumor necrosis factor $\alpha$ (TNF- $\alpha$ ), interleukin-6 (IL-6), CD62p, GMP-140, and neuron-specific enolase (NSE) were measured by an enzyme-linked immunosorbent assay with an automatic microplate reader (Thermo, USA).

Neurologic impairment before and after treatment was accessed using NIHSS scale with a score ranging from 0 to 42. High scores indicate severe neurologic impairment: 0-1: normal; 2-4: mild impairment; 5-15: moderate impairment; 16-20, moderate to severe impairment; over 21, severe impairment (18).

Cognitive function before and after treatment was evaluated using MMSE and MoCA. MMSE includes 19 items with a total score of 30 . MoCA has a total score of 30. Low scores indicate worst cognitive function (20).

Prognosis of all patients was evaluated at 1, 2, and 3 months after hospital discharge using the modified Rankin scale by the same physician, who was blinded to their treatment: 0 indicated that the patient was fully recovered; 1 , the patient showed symptoms but no significant dysfunction and was able to carry out daily activities; 2, the patient was mildly disabled without the need of assistance but with limited activities; 3, the patient was moderately disabled with the need of assistance for activities but was able to walk without the need of assistance; 4 , the patient was moderately disabled without the ability of independent walking and needed assistance from others to meet their life demands; 5 , the patient was severely disabled with the need of continuous care and assistance and suffered paralysis, incontinence, etc.; and 6 , the patient died. Patients with a Rankin scale score of 2 or below had a good prognosis and those with 3 and above had a poor prognosis.

Adverse reactions, such as cerebral hemorrhage, hematuria, skin allergy, nausea, and vomiting, during treatment were recorded (12).

\section{Statistical analysis}

SPSS 17.0 (IBM, USA) statistical software was used. Continuous variables are reported as means \pm SD. The data that conformed to normal distribution and homogeneity of variance were analyzed by the $t$-test; the independent-sample $t$-test was used for comparison among groups and the paired-sample $t$-test was employed for comparison before and after treatment within a group. The data that did not confirm to normal distribution and homogeneity of variance are reported as medians and quartiles and analyzed by the rank-sum test. Enumeration data were analyzed by Pearson chi-squared test. $\mathrm{P}<0.05$ indicated a statistically significant difference.

\section{Results}

\section{General data analysis}

There were no significant differences in gender, age, education background, body mass index, time from onset to admission, onset-to-treatment time, the TOAST classification, lesion location, and complications between the two groups (all $\mathrm{P}>0.05$, Table 1).

\section{Efficacy of treatments}

There were significant differences in the number of patients with recovery, remarkable improvement, improvement, and no change between the two groups (all $P<0.05)$. The total effectiveness rate in the observation group $(81.67 \%)$ was significantly higher than that $(61.67 \%)$ in the control group $(P<0.05$, Table 2$)$.

\section{Inflammatory factors and NSE after treatment}

There was no significant difference in inflammatory factors CRP, TNF- $\alpha$, IL-6, CD62p, GMP-140, and NSE before treatment between the two groups (all $P>0.05$ ). These indicators in the two groups were significantly lower after treatment compared with those before treatment (all $P<0.05)$, and the degree of decline in the observation group was higher than that in the control group $(P<0.001$, Table 3).

\section{NIHSS score after treatment}

There was no significant difference in the NIHSS score before treatment between the control group $(9.70 \pm 3.68)$ and the observation group $(9.64 \pm 3.62, \mathrm{P}>0.05)$. Compared with before treatment, the NIHSS scores in the control group $(5.57 \pm 1.48)$ and the observation group $(4.80 \pm 1.52)$ decreased after treatment $(P<0.05)$, however, the degree of decrease in the observation group was higher than that in the control group $(P<0.001)$.

\section{MMSE and MoCA scores after treatment}

There was no significant difference in the MMSE and MoCA before treatment between the two groups (both $P>0.05)$. The MMSE and MoCA were significantly improved after treatment compared to those before treatment (both $\mathrm{P}<0.05$ ). The MMSE and MoCA scores in the observation group were significantly higher than those in the control group after treatment (both $\mathrm{P}<0.05$, Table 4 and Figure 1).

\section{Modified Rankin scale scores 3 months after treatment}

There was no significant difference in modified Rankin scale scores 1 and 2 months after hospital discharge between the two groups $(P>0.05)$. The modified Rankin scale score 3 months after hospital discharge was significantly lower in 
Table 1. Comparison of general data and baseline data of patients with cute cerebral infarction treated with alteplase (observation group) or batroxobin (control group).

\begin{tabular}{|c|c|c|c|c|}
\hline & Observation group $(n=60)$ & Control group $(n=60)$ & $\chi^{2} / t$ & $P$ \\
\hline Gender (male:female) & $34: 26$ & $28: 32$ & 1.201 & 0.273 \\
\hline Age (years) & $68.5 \pm 7.1$ & $67.4 \pm 5.9$ & 0.692 & 0.492 \\
\hline Education background (years) & $12.50 \pm 3.85$ & $11.87 \pm 3.97$ & 0.627 & 0.533 \\
\hline $\mathrm{BMI}\left(\mathrm{kg} / \mathrm{m}^{2}\right)$ & $25.71 \pm 3.76$ & $25.59 \pm 4.28$ & 0.110 & 0.913 \\
\hline Time from onset to admission (h) & $3.12 \pm 0.76$ & $3.21 \pm 0.66$ & 0.234 & 0.786 \\
\hline Onset-to-treatment time $(\mathrm{h})$ & $3.78 \pm 0.87$ & $3.86 \pm 0.79$ & 0.256 & 0.756 \\
\hline Lesion location & & & 1.382 & 0.710 \\
\hline Brainstem & 7 & 5 & & \\
\hline Lobe & 13 & 10 & & \\
\hline Cerebellum & 6 & 9 & & \\
\hline Basal ganglia region & 34 & 36 & & \\
\hline TOAST classification & & & 0.651 & 0.957 \\
\hline Atherothrombosis & 38 & 36 & & \\
\hline Stroke of undetermined etiology & 11 & 12 & & \\
\hline Cardioembolism & 6 & 7 & & \\
\hline Small-vessel occlusion & 4 & 3 & & \\
\hline Stroke of other determined etiology & 1 & 2 & & \\
\hline Hyperlipidemia & 34 & 32 & 0.135 & 0.714 \\
\hline Hypertension & 40 & 34 & 1.269 & 0.260 \\
\hline Coronary heart disease & 20 & 24 & 0.574 & 0.449 \\
\hline Obesity & 20 & 26 & 1.269 & 0.260 \\
\hline Hyperhomocysteinemia & 44 & 48 & 0.745 & 0.388 \\
\hline Hyperuricemia & 38 & 44 & 1.386 & 0.239 \\
\hline
\end{tabular}

Data are reported as means \pm SD or number. BMl: body mass index.

Table 2. Comparison of efficacy based on the National Institutes of Health Stroke Scale of patients with acute cerebral infarction treated with alteplase (observation group) or batroxobin (control group).

\begin{tabular}{|c|c|c|c|c|c|}
\hline Group & Recovery & Remarkable improvement & Improvement & No change & Total effective rate \\
\hline Observation $(n=60)$ & $6(10.00)$ & $28(46.67)$ & $15(25.00)$ & $11(18.33)$ & $49(81.67)$ \\
\hline Control $(n=60)$ & $3(5.00)$ & $17(28.33)$ & $17(28.33)$ & $23(38.33)$ & $37(61.67)$ \\
\hline$\chi^{2}$ & \multicolumn{4}{|c|}{8.049} & 5.910 \\
\hline$P$ & \multicolumn{4}{|c|}{0.045} & 0.015 \\
\hline
\end{tabular}

Data are reported as number $(\%)$.

Table 3. Comparison of inflammatory factors and NSE before and after treatment of patients with cute cerebral infarction treated with alteplase (observation group) or batroxobin (control group).

\begin{tabular}{|c|c|c|c|c|c|c|c|c|}
\hline & \multicolumn{4}{|c|}{ Before treatment } & \multicolumn{4}{|c|}{ After treatment } \\
\hline & Observation group & Control group & $t$ & $P$ & Observation group & Control group & $t$ & $\mathrm{P}$ \\
\hline $\mathrm{CRP}(\mathrm{mg} / \mathrm{L})$ & $6.64 \pm 1.81$ & $6.90 \pm 1.84$ & 0.917 & 0.363 & $4.80 \pm 1.52^{\#}$ & $5.57 \pm 1.48^{\#}$ & 4.153 & $<0.001$ \\
\hline TNF- $\alpha(\mu \mathrm{g} / \mathrm{mL})$ & $124.51 \pm 18.22$ & $123.80 \pm 17.87$ & 0.154 & 0.878 & $85.01 \pm 15.90^{\#}$ & $102.66 \pm 16.12^{\#}$ & 4.269 & $<0.001$ \\
\hline IL-6 $(\mu \mathrm{g} / \mathrm{mL})$ & $351.37 \pm 37.86$ & $351.00 \pm 37.32$ & 0.038 & 0.969 & $273.27 \pm 25.64^{\#}$ & $318.34 \pm 29.73^{\#}$ & 6.287 & $<0.001$ \\
\hline CD62p $(\mathrm{pg} / \mathrm{mL})$ & $9.11 \pm 1.53$ & $9.14 \pm 1.59$ & 0.234 & 0.786 & $3.17 \pm 0.57^{\#}$ & $5.01 \pm 0.68^{\#}$ & 4.890 & $<0.001$ \\
\hline GMP-140 (ng/mL) & $50.89 \pm 10.12$ & $50.98 \pm 10.01$ & 0.192 & 0.896 & $27.65 \pm 6.52^{\#}$ & $36.79 \pm 7.61^{\#}$ & 6.902 & $<0.001$ \\
\hline NSE $(\mu \mathrm{g} / \mathrm{L})$ & $36.79 \pm 5.92$ & $37.32 \pm 5.76$ & 0.352 & 0.675 & $15.32 \pm 2.65^{\#}$ & $19.42 \pm 3.22^{\#}$ & 5.898 & $<0.001$ \\
\hline
\end{tabular}

Data are reported as means $\pm S D$. ${ }^{\#} \mathrm{P}<0.05$ before and after treatment compared within group. CRP: C-reactive protein; TNF- $\alpha$ : tumor necrosis factor $\alpha$; IL-6: interleukin-6; NSE: neuron-specific enolase. 
Table 4. Comparison of cognitive scores before and after treatment of patients with acute cerebral infarction treated with alteplase (observation group) or batroxobin (control group).

\begin{tabular}{|c|c|c|c|c|c|c|c|c|}
\hline & \multicolumn{4}{|c|}{ Before treatment } & \multicolumn{4}{|c|}{ After treatment } \\
\hline & Observation group & Control group & $t$ & $\mathrm{P}$ & Observation group & Control group & $t$ & $P$ \\
\hline MMSE & $25.42 \pm 1.22$ & $25.37 \pm 1.16$ & 0.108 & 0.914 & $27.83 \pm 0.91^{\#}$ & $25.70 \pm 1.80$ & 5.201 & $<0.001$ \\
\hline MoCA & $22.50 \pm 1.94$ & $22.20 \pm 2.35$ & 0.538 & 0.592 & $23.73 \pm 2.03^{\#}$ & $22.23 \pm 2.18$ & 2.759 & 0.008 \\
\hline
\end{tabular}

Data are reported as means \pm SD. ${ }^{\#} \mathrm{P}<0.05$ before and after treatment compared within group. MMSE: mini-mental state examination; MoCA: Montreal Cognitive Assessment.

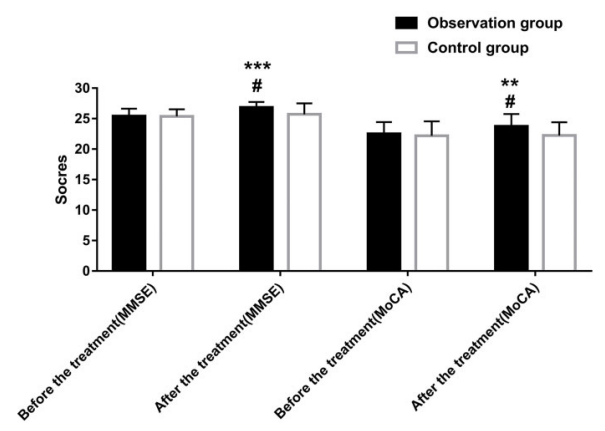

Figure 1. Comparison of cognitive scores after treatment. Data are reported as means $\pm \mathrm{SD}$. ${ }^{* *} \mathrm{P}<0.01$, ${ }^{* *} \mathrm{P}<0.001$ compared with the control group; ${ }^{\#} \mathrm{P}<0.05$ compared with before treatment (independent-sample $t$-test and the paired-sample $t$-test). MMSE: mini-mental state examination; MoCA: Montreal Cognitive Assessment.

the observation group than in the control group $(\mathrm{P}<0.01)$. Three months after discharge, the modified Rankin scale scores in the two groups were lower than those at 1 month; moreover, the percentage of patients who had 0-1 scores in the observation group was significantly higher than those in the control group $(\mathrm{P}<0.05$, Table 5).

\section{Adverse reactions}

No patient died during treatment in the two groups. In the observation group, there was 1 patient with asymptomatic hemorrhagic transformation (evaluated by NINDS criteria), 1 patient with hematuria, and 1 patient with nausea and vomiting, with a total incidence rate of adverse reactions of $5 \%(3 / 60)$. In the control group, there were 2 patients with asymptomatic hemorrhagic transformation (evaluated by NINDS criteria) and 2 patients with nausea and vomiting, with a total incidence rate of adverse reactions of $6.67 \%(4 / 60)$. There was no significant difference in the total adverse reaction incidence rate between the two groups $\left(\chi^{2}=0.154, P=0.695\right)$.

\section{Discussion}

In the early stage of $\mathrm{ACl}$, thrombolytic therapy within the time window of $4.5 \mathrm{~h}$ has positive and important significance for prognosis (21). Early thrombolytic therapy can remove vascular blockage as soon as possible, which is beneficial for the reduction of cerebral infarction area and the reconstruction of ischemic circulation to save the ischemic penumbral area (9). Batroxobin, an anti-fibrinogen agent, has the pharmacological effect of reducing blood viscosity, decomposing fibrinogen, inducing thrombolysis and inhibiting thrombosis (22). A large amount of evidence proves that batroxobin is safe and effective in treating $\mathrm{ACl}$, and it is recommended by the 2014 Guidelines for Diagnosis and Treatment of Acute Ischemic Stroke in China $(17,23)$.

Alteplase is a new type of thrombolytic agent, which belongs to gene recombinant thrombolytic drug line. The drug can combine with fibrin after it enters the blood, then activates fibrinogen and promotes the degradation of plasma fibrinogen, thus promoting a thrombolytic effect, and the vessel is rapidly recanalized. Alteplase has become the first choice for the treatment of $\mathrm{ACl}$ in European and American countries $(10,24,25)$. In our study, patients with alteplase treatment had a higher decrease in the NIHSS score than those with batroxobin treatment, which is consistent with the results from previous studies $(12,13)$, indicating that patients could obtain more benefits from alteplase than batroxobin.

A Chinese study has shown that thrombolysis with 0.9 $\mathrm{mg} / \mathrm{kg}$ alteplase has significant advantages and does not increase the risk of intracranial hemorrhage (26). In this study, we found that among $\mathrm{ACl}$ patients, total effective rate of alteplase was $81.67 \%$, which was significantly higher than that of batroxobin $(61.67 \%)$, and no difference was found in the incidence of adverse reaction (hemorrhage) between the two drugs, which was consistent with the above study.

Inflammatory factors play an important role in the development of $\mathrm{ACl}$. CRP is a commonly used clinical indicator, which is synthesized in the liver under the mediation of inflammatory factors like IL-6 (27). Previous study has shown that CRP can reflect the degree of neurological impairment in $\mathrm{ACl}$ patients (28). TNF- $\alpha$ has dual biological effects. It not only has an anti-infection effect when its concentration is low in the human body, but also plays a role in the regulation of tissue repair of inflammatory responses. The body's immunity is 
Table 5. Comparison of modified Rankin scale scores of patients with acute cerebral infarction treated with alteplase (observation group) or batroxobin (control group).

\begin{tabular}{|c|c|c|c|c|}
\hline & Observation group & Control group & $\chi^{2} / t$ & $\mathrm{P}$ \\
\hline At 1 month after discharge & $2.7 \pm 0.5$ & $2.9 \pm 0.7$ & 0.895 & 0.126 \\
\hline Rate of patients with scores $0-1$ at 1 month after discharge & $11(18.33)$ & $8(13.33)$ & 0.563 & 0.453 \\
\hline At 2 months after discharge & $2.3 \pm 0.5$ & $2.7 \pm 0.7$ & 0.912 & 0.118 \\
\hline Rate of patients with scores $0-1$ at 2 months after discharge & $16(26.67)$ & $10(16.67)$ & 1.768 & 0.184 \\
\hline At 3 months after discharge & $1.9 \pm 0.3^{* *}$ & $2.5 \pm 0.7^{*}$ & 2.796 & 0.007 \\
\hline Rate of patients with scores $0-1$ at 3 months after discharge & $25(41.67)$ & $14(23.33)$ & 4.596 & 0.032 \\
\hline
\end{tabular}

Data are reported as means $\pm S D$ or number and $(\%)$. ${ }^{*} \mathrm{P}<0.05,{ }^{* *} \mathrm{P}<0.01$ compared with the modified Rankin scale score at 1 month after discharge.

destroyed when the concentration of TNF- $\alpha$ increases, which could lead to the activation of neutrophils, increased phagocytosis of white blood cells, and the promotion of inflammatory factors secretion, thereby increasing vascular permeability (29). In addition, TNF- $\alpha$ plays an important role in the development and progression of $\mathrm{ACl}$, and its content in vivo is positively correlated with cerebral infarction volume and nerve function injury (30). TNF- $\alpha$ can also promote thrombosis and inhibit fibrinolysis (31). IL-6 starts to increase when the brain tissue is damaged, and an appropriate increase of IL-6 is conducive to the functioning of the brain tissue, while an excessive increase will cause further brain tissue damage (32). Previous research has found that alteplase can inhibit the inflammatory response lowering the incidence of hemorrhagic transformation and reducing the risk of hemorrhage after thrombolysis (33). CD62P is a key factor reflecting platelet activation, and GMP140 mediates platelet activation in vascular endothelial injury and promotes the progression of cerebral infarction (34). NSE is the specific key enzyme of glycolysis, which can peak in serum and cerebrospinal fluid $8 \mathrm{~h}$ after brain tissue injury (35). The blood-brain barrier permeability changes due to brain tissue damage in brain diseases, and NSE can enter the blood, thus significantly increasing its content in the blood (36). In this study, alteplase had better effects on reducing inflammatory factors and relieving nerve injury than batroxobin, which was consistent with the above results.

\section{References}

1. Arba F, Inzitari D, Ali M, Warach SJ, Luby M, Lees KR. Small vessel disease and clinical outcomes after IV rt-PA treatment. Acta Neurol Scand 2017; 136: 72-77, doi: 10.1111/ ane.12745.

2. Sagnier S, Galli P, Poli M, Debruxelles S, Renou P, Olindo S, et al. The impact of intravenous thrombolysis on outcome of patients with acute ischemic stroke after 90 years old. BMC Geriatr 2016; 16: 156, doi: 10.1186/s12877-016-0331-1.

3. Wahlgren N, Moreira $T$, Michel $P$, Steiner $T$, Jansen $O$, Cognard $\mathrm{C}$, et al. Mechanical thrombectomy in acute ischemic stroke: Consensus statement by ESO-Karolinska
Thrombolysis with alteplase can significantly alleviate the hypoxia of brain tissue and reduce the damage of neurogliocytes and neurons, reducing the damage of brain tissue (37). MMSE and MoCA are common evaluation tools to measure the cognitive function of patients $(18,20)$. In this study, alteplase had better effects on reducing brain injury and improving cognitive function than batroxobin, which was consistent with the above research results. Rankin scale is an important indicator to evaluate the prognosis of $\mathrm{ACl}$ (38). We found that the score of the modified Rankin scale was lower in patients treated with alteplase than that in those treated with batroxobin 3 months after treatment, suggesting that alteplase could improve the prognosis of $\mathrm{ACl}$ patients.

However, the sample size of this study was small, which needs to be further expanded. Additionally, the observing time was short, so the follow-up time needs to be further lengthened. Patients with contraindication for thrombolysis who could not tolerate thrombolytic therapy were not enrolled in this study for control observation. Therefore, these patients should be included in future controlled observational studies to determine the effect of thrombolytic therapy in improving prognosis.

In conclusion, intravenous thrombolytic therapy with alteplase for $\mathrm{ACl}$ enhanced clinical improvement, reduced inflammatory response and brain injury, and improved cognitive function, which is worthy of further clinical application and research.
Stroke Update 2014/2015, supported by ESO, ESMINT, ESNR and EAN. Int J Stroke 2016; 11: 134-147, doi: 10.11 77/1747493015609778.

4. Juan Didier PG, Edgardo Jorge EV, Martinez HR. Epidemiology of ischemic strokes in elderly. Int J Stroke 2016; 11: NP54, doi: 10.1177/1747493016632256.

5. Teh WL, Abdin E, Vaingankar JA, Seow E, Sagayadevan V, Shafie S, et al. Prevalence of stroke, risk factors, disability and care needs in older adults in Singapore: results from the WiSE study. BMJ Open 2018; 8: e020285, doi: 10.1136/ bmjopen-2017-020285. 
6. Gan Y, Wu J, Zhang S, Li L, Yin X, Gong Y, et al. Prevalence and risk factors associated with stroke in middle-aged and older Chinese: a community-based cross-sectional study. Sci Rep 2017; 7: 9501, doi: 10.1038/s41598-01709849-z.

7. GBD 2016 Couses of Death Collaborators. Global, regional, and national age-sex specific mortality for 264 causes of death, 1980-2016: a systematic analysis for the Global Burden of Disease Study 2016. Lancet 2017; 390: 1151-1210, doi: 10.1016/S0140-6736(17)32152-9.

8. Kim JS, Kim YJ, Lee KB, Cha JK, Park JM, Hwang Y, et al. Low- versus standard-dose intravenous alteplase in the context of bridging therapy for acute ischemic stroke: a Korean ENCHANTED Study. J Stroke 2018; 20: 131-139, doi: 10.5853/jos.2017.01578.

9. Zhao XX, Mu LC, MA LF, Department N. Clinical study on intravenous thrombolysis with recombinant tissue-type plasminogen activator for acute cerebral infarction [in Chinese]. Chinese Journal of Evidence-Based Cardiovascular Medicine 2016, <http://en.cnki.com.cn/Article_en/ CJFDTOTAL-PZXX201604033.htm >.

10. Powers WJ, Rabinstein AA, Ackerson T, Adeoye OM, Bambakidis NC, Becker K, et al. Guidelines for the Early Management of Patients With Acute Ischemic Stroke: 2019 Update to the 2018 Guidelines for the Early Management of Acute Ischemic Stroke: A Guideline for Healthcare Professionals From the American Heart Association/American Stroke Association. Stroke 2019; 50: e344-e418, doi: 10.1161/STROKEAHA.118.022606.

11. Huang YM, Huang SG, Emergency DO. Effect of batroxobin treatment time on the cerebrovascular reserve function in patients with acute cerebral infarction [in Chinese]. Medical Recapitulate 2016, < http://en.cnki.com.cn/Article_en/CJFD Total-YXZS201613044.htm > .

12. Ingall TJ, O'Fallon WM, Asplund K, Goldfrank LR, Hertzberg VS, Louis TA, et al. Findings from the reanalysis of the NINDS tissue plasminogen activator for acute ischemic stroke treatment trial. Stroke 2004; 35: 2418-2424, doi: 10.1161/01.STR.0000140891.70547.56.

13. Hacke W, Donnan G, Fieschi C, Kaste M, von Kummer R, Broderick JP, et al. Association of outcome with early stroke treatment:pooled analysis of ATLANTIS, ECASS, and NINDS rt-PA stroke trials. Lancet 2004; 363: 768-774, doi: 10.1016/S0140-6736(04)15692-4.

14. Nathaniel TI, Cochran T, Chaves J, Fulmer E, Sosa C, Yi S, et al. Co-morbid conditions in use of recombinant tissue plasminogen activator (rt-PA) for the treatment of acute ischaemic stroke. Brain Inj 2016; 30: 1261-1265, doi: 10.1080/02699052.2016.1186840.

15. Atik I, Kozaci N, Beydilli I, Avci M, Ellidag H, Kesapli M. Investigation of oxidant and antioxidant levels in patients with acute stroke in the emergency service. Am J Emerg Med 2016; 34: 2379-2383, doi: 10.1016/j.ajem.2016.08.062.

16. Lu K, Xu X, Cui S, Wang F, Zhang B, Zhao Y. Serum neuron specific enolase level as a predictor of prognosis in acute ischemic stroke patients after intravenous thrombolysis. J Neurol Sci 2015; 359: 202-206, doi: 10.1016/j.jns.2015. 10.034 .
17. Chinese Society of Neurology. Neurology cerebrovascular disease study group of the Chinese Medical Association. Guidelines for diagnosis and treatment of acute ischemic stroke in China 2014 [in Chinese]. Chin J Neurol 2015; 48: 246-257, < http://med.wanfangdata.com.cn/Paper/Detail/ PeriodicalPaper_zhsjk201504002>.

18. Lyden P. Using the National Institutes of Health Stroke Scale: a cautionary tale. Stroke 2017; 48: 513-519, doi: 10.1161/STROKEAHA.116.015434.

19. Adams HP Jr, Bendixen BH, Kappelle LJ, Biller J, Love BB, Gordon DL, et al. Classification of subtype of acute ischemic stroke. Definitions for use in a multicenter clinical trial. TOAST. Trial of Org 10172 in Acute Stroke Treatment. Stroke 1993; 24: 35-41, doi: 10.1161/01.STR.24.1.35.

20. Ciesielska N, Sokolowski R, Mazur E, Podhorecka M, PolakSzabela A, Kedziora-Kornatowska K. Is the Montreal Cognitive Assessment (MoCA) test better suited than the Mini-Mental State Examination (MMSE) in mild cognitive impairment $(\mathrm{MCl})$ detection among people aged over 60 ? Meta-analysis. Psychiatr Pol 2016; 50: 1039-1052, doi: 10.12740/PP/45368.

21. Bang OY. Clinical trials of adult stem cell therapy in patients with ischemic stroke. J Clin Neurol 2016; 12: 14-20, doi: 10.3988/jcn.2016.12.1.14.

22. Jiang HF, Huang J, Huang P, Qiu XH, Qu QF. Risk factors of hemorrhagic transformation following defibrase therapy in patients with acute cerebral infarction [in Chinese]. Chin $J$ Neuromed 2016; 15: 11-14, < http://d.wanfangdata.com.cn/ periodical/zhsjyxzz201601003>.

23. Huang YN. Comparison of the efficacy and safety between urokinase and batroxobin in treatment of acute ischemic stroke [in Chinese]. Chinese Journal of Geriatric Heart Brain \& Vessel Diseases 2006, < http://d.wanfangdata.com. cn/periodical/ChIQZXJpb2RpY2FsQ0hJTmV3UzIwMjAxM j14EhR6aGxueG54Z2J6ejlwMDYwMjAxMBolaXVxbzNrYXc $\% 3 \mathrm{D}>$.

24. Bonaventura A, Montecucco F, Dallegri F. Update on the effects of treatment with recombinant tissue-type plasminogen activator (rt-PA) in acute ischemic stroke. Expert Opin Biol Ther 2016; 16: 1323-1340, doi: 10.1080/14712598. 2016.1227779.

25. European Stroke Organisation (ESO) Executive Committee; ESO Writing Committee. Guidelines for management of ischaemic stroke and transient ischaemic attack 2008. Cerebrovasc Dis 2008; 25: 457-507, doi: 10.1159/00013 1083.

26. Liao XL, Wang YL, Pan YS, Wang CJ, Zhao XQ, Wang CX, et al. Comparison of the efficacy and safety of acute ischemic stroke patients receiving different dose intravenous alteplase [in Chinese]. Chin J Stroke 2014, <http://d. wanfangdata.com.cn/periodical/ChIQZXJpb2RpY2FsQOhJT mV3UzIwMjAxMj14Eg96Z3p6enoyMDE0MDcwMDQaCDQz Znl4aHN2>.

27. Zhang Q, Qian G, Ding Z. Xuemaitong granules attenuate carotid atherosclerosis by decreasing the expression of CD14 + CD16 + monocytes, IL-6, TNF-alpha, and hsCRP. Genet Mol Res 2014; 13: 7519-7527, doi: 10.4238/2014. September.12.19. 
28. Yuan T, Wang M, Zhao X, Ren S. The correlation between neutrophil to lymphocyte ratio,high-sensitivity C-reactive protein and acute cerebral infarction [in Chinese]. Chin $J$ Neuroimmunol Neurol 2016, <http://d.wanfangdata.com.cn/ periodical/ChIQZXJpb2RpY2FsQ0hJTmV3UzIwMjAxMjl4 EhV6Z3NqbXI4aHNqYngyMDE2MDMwMTMaCDdwd2xtb $201>$.

29. Camelo S, Lafage M, Galelli A, Lafon M. Selective role for the p55 Kd TNF-alpha receptor in immune unresponsiveness induced by an acute viral encephalitis. J Neuroimmunol 2001; 113: 95-108, doi: 10.1016/S0165-5728(00) 00427-6.

30. Zhang Y, Dai Q, Li C, Neurology DO, Hospital JC. Correlation analysis between neural function recovery and inflammatory factors in patients with acute stroke [in Chinese]. Anhui Medical Journal 2016, <http://d.wanfang data.com.cn/periodical/ChIQZXJpb2RpY2FsQOhJTmV3Uzl wMjAxMj14Eg1haHI4MjAxNjA1MDIxGgh2bGd1MjFmYQ\%3 $\mathrm{D} \% 3 \mathrm{D}>$.

31. Chen L, Yang Q, Ding R, Liu D, Chen Z. Carotid thickness and atherosclerotic plaque stability, serum inflammation, serum MMP-2 and MMP-9 were associated with acute cerebral infarction. Exp Ther Med 2018; 16: 5253-5257.

32. Hou X, Manli TU, Gang YU, Neurology DO, Hospital FA, University NM. Expressions of serum interleukin-6 and interleukin-18 in patients with acute cerebral infarction and vascular cognitive impairment [in Chinese]. Jiangsu Medical Journal 2016, < http://en.cnki.com.cn/Article_en/CJFDTotalYIYA201603013.htm >.
33. Patil MS, Jayaraman MV, Ahn SH. Vascular closure devices in stroke patients receiving tissue plasminogen activator: A retrospective analysis from an academic tertiary medical center and a teaching community hospital stroke database. Clin Neurol Neurosurg 2017; 157: 22-24, doi: 10.1016/ j.clineuro.2017.03.023.

34. Sabbaghziarani F, Mortezaee K, Akbari M, Kashani IR, Soleimani M, Hassanzadeh G, et al. Stimulation of neurotrophic factors and inhibition of proinflammatory cytokines by exogenous application of triiodothyronine in the rat model of ischemic stroke. Cell Biochem Funct 2017; 35: 50-55, doi: 10.1002/cbf.3244.

35. Sun J, Li J, Cheng G, Sha B, Zhou W. Effects of hypothermia on NSE and S-100 protein levels in CSF in neonates following hypoxic/ischaemic brain damage. Acta Paediatr 2012; 101: e316-e320, doi: 10.1111/j.1651-2227.2012.02679.x.

36. White GS, Symmes K, Sun P, Fang Y, Garcia S, Steiner C, et al. Reemergence of St. Louis Encephalitis Virus, California, 2015. Emerg Infect Dis 2016; 22: 2185-2188, doi: 10.3201/eid2212.160805.

37. Bracard S, Ducrocq X, Mas JL, Soudant M, Oppenheim C, Moulin T, et al. Mechanical thrombectomy after intravenous alteplase versus alteplase alone after stroke (THRACE): a randomised controlled trial. Lancet Neurol 2016; 15: 11381147, doi: 10.1016/S1474-4422(16)30177-6.

38. Muir KW, Weir CJ, Murray GD, Povey C, Lees KR. Comparison of neurological scales and scoring systems for acute stroke prognosis. Stroke 1996; 27: 1817-1820, doi: 10.1161/ 01.STR.27.10.1817. 